\title{
Enhancement of economic efficiency of transport performance using multi-criteria estimation
}

\author{
M. Yu. Karelina \\ Moscow Automobile and Road State Technical University \\ Leningradsky prospect 64, 125319 Moscow \\ Russia \\ karelinamu@mail.ru \\ V.V. Moiseev \\ Shukhov Belgorod State Technological University, 46 \\ Kostyukova St., Belgorod, 308012, \\ Russia, \\ din_prof@mail.ru
}

\begin{abstract}
The article outlines the approach to the solution of the multi-criteria economic task of choosing a transport for certain operating conditions based on vector optimization methods and linear programming. Full disclosure of the potential capabilities of the transport system in the economy sector requires the formation and management of automobile parks, taking into account the specific features of production. Therefore, there is a need to evaluate the efficiency of transport operation according to a number of properties of the complex state of its quality. The solution of the task should be based on the determination of the importance of particular economic indicators for each property, defined as a separate quality criterion. To do this, it is necessary to form a system of indicators of vehicle quality properties and to develop a formalized apparatus for obtaining the weight of each criterion in the form of a multi-criteria evaluation method for the complex property of a vehicle. The solution of this task will make it possible to increase the economic efficiency of using the AIC transport parks by realizing fully the potential capabilities of each transport unit, as well as the entire fleet, and will increase the possibility of managing the peak volumes of transportation by a smaller number of vehicles, reducing the need for transport involved. In turn, this will entail a reduction in the share of transportation costs in production costs for domestic production and will result in significant cost savings. As theoretical and methodological issues related to the multi-criteria assessment of the quality of vehicles used in the economy sector have not been fully developed yet, the need for such studies is urgent, including creation on their basis of methods for formation and management of the park of enterprises of the economy sector.
\end{abstract}

Keywords-economy sector; multi-criteria evaluation; management; system of operational properties.

\section{INTRODUCTION}

The State Program for the Development of Agriculture and Regulation of Agricultural Products, Raw Materials and Foodstuffs for 2013-2020 covers the entire range of directions for the development of the agro-industrial complex in the Russian Federation. The current trend in the growth in the production of agricultural output requires new approaches to solving the transport problems of the agro-industrial complex (AIC) that contribute to improving the quality of transport services in its branches.

\author{
A. V. Terentyev \\ Moscow Automobile and Road State Technical University \\ Leningradsky prospect 64, 125319 Moscow \\ Russia \\ aleksej.terentev.67@bk.ru \\ V.V.Stroev \\ Kutafin Moscow State Law University \\ (MSAL), Sadovaya-Kudrinskaya St., 9, Moscow, \\ Russia, 123995, Vstroev@yandex.ru
}

The leading place in the transport system of the agroindustrial complex is motor transport. This kind of transport is mainly used on external freight traffic, which means roads between a certain economy unit and the road infrastructure surrounding it. On-farm delivery of goods in the main branches of production in agricultural organizations often supposes use of tractors. Occupying the largest share in the structure of cargo transportation of the agrarian sector, road transport is an important component in the system of transport servicing of the agro-industrial complex. Statistics show that the level of power availability of the agro-industrial complex by machine-tractor means in different regions of the Russian Federation is distributed unevenly. In a number of regions, the main problems of road transport in the agro-industrial complex currently are as follows: a high degree of wear of rolling stock (more than $80 \%$ ), a considerable average life of automotive equipment (more than 12 years), a constant increase in costs for current repairs and maintenance of cars, and, as a consequence, an increase in the number of failures and downtimes of vehicles for technical reasons. This current state of the machine and tractor fleet of the agro-industrial complex is not able to ensure the implementation of promising areas of scientific and technological development of the agro-industrial complex of the Russian Federation for the period until 2030, guaranteeing food security and allowing Russia to become a global supplier of food products of high processing level. According to various estimates, in order to ensure the country's food independence and performance of work in optimal time, it will be necessary to have an energy supply per 1 hectare of arable land, which amounts to at least $3 \mathrm{hp}$ (in fact, at the present time - $1.5 \mathrm{hp}$, while in the EU it amounts to 4.0-4.5 hp, and in the USA - to $8.5 \mathrm{hp}$ ). Today, the most suitable option for the agrarian and industrial complex of the Russian Federation is the machine and tractor fleet, which includes 850-900 thousand units (taking into account the need to develop 30 million hectares, and without this - 630 thousand units) [1].

Full disclosure of the potential capabilities of the transport system of the agro-industrial complex requires the formation of automobile parks, considering the specific features of agricultural production and the conformity of the characteristics of vehicle designs to them. The transport parks formed on this 
basis must meet the requirements of both the conditions for the production of agricultural enterprises and the operating environment as a whole. Trends in developing the design of modern vehicles involve the provision of such properties of the car as environmental, constructive and fire safety, which is definitely relevant while operating these vehicles in the agroindustrial complex [2]. It can be stated that a number of qualitative properties of cars are the criteria, the performance indicators of which vary in time with the increase in the lifetime of vehicles.

Theoretical research determines that some of the most important properties of the car, for example, efficiency, productivity, reliability, change exponentially in operating time [3]:

$$
P(t)=P_{0} \cdot e^{-\beta t},
$$

where $P_{0}$ is the value of the parameter at the beginning of operation, $\beta$ - the coefficient characterizing the change of $P$ according to the time of operation of the car.

At present, as a rule, the rate of change of individual groups of quality indicators is averaged, both for a single vehicle and for their total amount. According to [4], regarding the intensity of the changes, complex and unit indicators are divided into three main groups:

1. Those that have an insignificant rate of change (from 0.9 to 1.1). This group includes indicators that provide such parameters of vehicle operation efficiency as: the coefficient of technical readiness, the output ratio, the specific downtime in maintenance and repair.

2. Those that have a significant rate of change (from 1.5 to 5.0). The second group includes indicators that characterize the reliability of vehicle components and units, and thus ensure the performance and technical safety of the car.

3 . Those that have a rate leading to a change in the indicator within a range close or exceeding the order of the initial value (from 7 to 20 and more). The third group of indicators characterizes basically the essence of the qualitative changes that occur when the product is aging (the cost of parts being replaced, the consumption of spare parts). It is more responsible for the environmental safety and constructive safety of the vehicle considering the toughening of the requirements of vehicle operating environment.

Therefore, the development of methods for assessing the quality of vehicles for a number of quality properties that are required in agro-industrial production is an urgent task in the face of increasing requirements for the efficiency of their operation [5]. The scientific approach to solving this problem dictates the need to make an optimal decision, not one at a time, but at once on a number of quality criteria, that is, it requires a multi-criteria evaluation.

\section{PROBLEM STATEMENT}

At present, a significant weight in solving practical problems of the agrarian and industrial complex of the Russian Federation acquires the solution of certain optimization problems. The methods of mathematical modeling of transport problems developed for modern conditions should allow constructing algorithms that provide an optimal solution for the implementation of applied problems and have a wide scope in the presence of minimal information about the states of the research object. Let us enumerate the main directions of development of methods for obtaining qualitative assessments of objects or processes that are actualized in this study:

1) vector optimization methods for solving multi-criteria tasks;

2) tasks with a solution based on expert assessments;

3) tasks based on the principles of ranking;

4) tasks of optimizing the parameters of systems in conditions of incompleteness of information about the object of research.

A sufficient number of studies have been devoted to methods for evaluating the efficiency of vehicle operation for a number of qualitative properties $[6,7,8,9]$. They rightly note two main difficulties in solving the task stated.

1. The difficulty of choosing the nomenclature of vehicle quality indicators. Depending on the type, operating conditions and purpose of the vehicle, the number of estimated indicators can be quite large. Naturally, with their increase, the laboriousness of their cumulative assessment increases, while the subjectivism of the decision to be taken is also increasing. Therefore, it is recommended to enter only the most significant indicators from the point of view of vehicle explants due to operating conditions, regulatory requirements, etc., that is, the need to identify the priority of evaluation criteria is determined.

2. Another problem of assessing the properties of cars is the procedure for correctly comparing alternative property options (it is impossible to do it by one single indicator only), so a multi-criteria task of efficiency evaluation is formed. In this case the most vulnerable point in terms of the objectivity of the decision is to determine the weight coefficients of relative importance of the criteria in question.

To determine weight coefficients of relative importance with the help of expert assessments, a sufficiently diverse and wide range of methods has been developed. For example, in [10] the following methods are considered: the method of preference vectors; the method of discrete expert curves; the paired comparison method; method of midpoint, etc. Ultimately, the methods examined and enumerated are aimed at enabling the decision-maker to make informed decisions based on the experience, knowledge and intuition of specialists. Thus, the lack of information is compensated for by formalized knowledge of experts, which increases for subjectivism in decision-making. With this goal in mind - receipt and processing of such information - an independent discipline connected with theory and practice of expert assessments began to develop. As a rule, for the work on the method of expert assessment, a working group is set up, which organizes, on behalf of the decision maker, the activities of experts united in an expert commission. Expert assessments are applied either when choosing one option from many criteria or when determining the weight of each of the criteria proposed in the study. Expert assessments can be either individual or collective. As already noted, there are many methods for obtaining expert assessments. In some of them, work with each expert is carried out individually, in other cases all the experts get grouped together. In some methods, the number of experts is fixed and does not change with time, in others the number of experts 
increases in the process of researching a particular process, for example, if the "snowball" method is applied.

Methods of expert assessments differ not only in the number of experts involved, but also depending on the type of tasks to be accomplished and the objectives of the study:

1) the "Delphi" method is used to predict the results of scientific and technological development;

2) the "Scenarios" method is used for socio-economic or environmental expert forecasting;

3) the "Brainstorming" method is designed to develop new ideas: experts offer their opinion and, in the course of discussion, find a compromise solution.

From a mathematical point of view, it is easier to analyze expert assumptions if they have a numerical form, thereby obtaining a compromise and a coordinated solution, which, as a rule, satisfies all experts. In practice, two main measurement scales are used: the quantitative scale and the qualitative one. Most often opinions of experts are expressed on a quantitative scale, and in this case the expert can claim that one indicator is more important than another one. In a qualitative scale (ordinal scale or scale of names), numerical values are used as "marks" reflecting the significance of the indicator, therefore, the use of this scale for solving multi-criteria problems is not always objective. It is not infrequently suggested that it should be determined whether the agreed opinion is the mean one of all the assumptions of the experts and that the arithmetic mean of expert assessments should be taken as the most reasonable one for solving multi-criteria problems. At the same time it is considered that the decision makers possess information about the competence of the experts themselves and form their own assessment for the same experts. As a result, we obtain a hierarchy, where the evaluation of the experts of the decision maker is first presented, and then expert assessments of the criteria are given directly in the multi-criteria choice problem and further the procedure for determining the compromise weight vector of the criteria is considered, taking into account both the experts' assumptions and also the opinion of the decision maker about the experts themselves. Thus, a task is formed with a hierarchy of several levels, which in itself is a significant difficulty for finding the optimal solution. In general, it can be concluded that there is no uniform method of solving multi-criteria problems based on expert assessments, considering the fact that such uniform method is the most effective in the opinion of most specialists. Therefore, there is a problem of developing a mathematical apparatus that would allow us to find a solution to multi-criteria problems in a dynamic system regardless of the participation of a human being.

\section{RESEARCH QUESTIONS}

It might be more promising to consider the application of multi-criteria optimization methods for solving the problems stated above, and these methods can be conditionally divided into two large groups:

Methods that are less based on expert assessment to obtain values of weights or their priority of importance. The solution of this task, as a rule, is based on determining the importance of selected individual indicators for each criterion, based on an intuitive representation of the comparative importance of the criteria by experts. For this purpose, many different methods have been developed, which have their own advantages and disadvantages: the method of ranking; the method of assigning points; the method of hierarchy analysis (MHA) [11,12], etc.

If we analyze and compare the methods listed above, then we should definitely highlight the MHA method. Its use does not require an expert survey, that is, it is not necessary to know the specific weight coefficients, but we must answer the question of how many times one indicator is more important than another one in order to build a matrix of paired comparisons, and then check its consistency, which is quite laborious. Although here are approaches to simplify this procedure, too. The main drawback of the methods of this group is a significant degree of subjectivism of the decision, because the person (the expert) is not really able to objectively assign the correct numerical weights to the criteria.

Methods based on special procedures for obtaining weight coefficients or formal methods for obtaining weight coefficients. Let us list some of them: the method of concessions, the ideal point method, the method of collapsing criteria, the Fishburn estimate, the Laplace criterion, etc. [13]. Among the methods listed above, the most simple and not requiring any complicated additional studies method is that of calculating weight coefficients by the Fishburn formulas. The main disadvantage inherent in these methods to some extent is that between the private indicators a strictly formalized connection is established (the $s$-th indicator is recognized more important than the $t$-th $p$ times). Obviously, such conclusion is subjective and most often cannot be justified [14].

\section{PURPOSE OF THE STUDY}

Thus, it is possible to form the research objective as the development of a method for multi-criteria quality assessment of a vehicle that meets the following basic requirements:

- the method must take into account the priority of the criteria;

- the solution obtained should be the maximum possible in a quantitative assessment, taking into account the performance indicators according to the established quality criteria;

- the apparatus for obtaining the weight of the criterion must be formalized, that is, objective in terms of obtaining the weight of a separate criterion for each individual solution.

\section{METHODS OF RESEARCH}

These factors force us to look for possible ways of solving this problem. Let us consider the possibility of applying the zoning method [14] to solve multi-criteria tasks. From the general theory of operations research, it is known that any possible solutions are stable with respect to changes in the initial information. This means that the goal-directed refinement of the necessary information (for example, setting the priority of significance between the criteria) makes it much easier to find the optimal solution. The essence of the zoning method consists in splitting the set of vectors characterizing the state of the research environment into subsets of dominance of individual actions, and the zoning operation is an inverse parametric problem of linear programming. Therefore, in order 
to increase the reliability of the decisions made, it is extremely important to choose a zoning method.

These provisions make it possible to form a zoning method for solving multi-criteria tasks, based on the partition of the set of possible states of the external environment of the study, according to the hierarchical relationship between the probabilities of their appearance. In this case, the probabilities of the states of the research environment are, in their sense, adequate to the coefficients of relative importance of the criteria in multi-criteria tasks.

Let us explain the essence of the proposed method by introducing the following notations:

$m-$ the number of vehicle types;

$n$ - the number of properties (criteria) by which vehicles are evaluated;

$a_{i j}$ - the numerical value $j$-th indicator for the $i$-th type of a vehicle, $i=\overline{1, m}, j=\overline{1, n}$;

Then the matrix of possible efficiencies for different solutions has the following form:

$$
\left\|a_{i j}\right\|=\left(\begin{array}{cccc}
a_{11} & a_{12} & \cdots & a_{1 n} \\
a_{21} & a_{22} & \cdots & a_{2 n} \\
\ldots & & & \cdots \\
a_{m 1} & a_{m 2} & \cdots & a_{m n}
\end{array}\right) .
$$

Distribution of coefficients of relative importance of efficiency criteria is subject to the following constraints:

$$
0 \leq \mathrm{c}_{j} \leq 1, \quad j=\overline{1, n}, \quad \sum_{j=1}^{n} \mathrm{c}_{j}=1,
$$

that is, it is determined by a set of $(n-1)$ independent variables.

In this case, the set of distributions of coefficients of relative importance will correspond to the field of distributions in the form of a rectangular unit hypertetrahedron in measurement space $(n-1)$.

In a Cartesian coordinate system $\mathrm{c}_{1}, \mathrm{c}_{2} \ldots, \mathrm{c}_{n-1}$, such hypertetrahedron is the result of the intersection of a positive hyperoctant by a hyperplane that cuts off a segment equal to one on each of the coordinate axes:

$$
\sum_{j=1}^{n-1} c_{j}
$$

Let us consider the situation when there are grounds for the properties of a vehicle in the sequence at which the condition of their priority is met:

$$
\mathrm{c}_{1} \geq \mathrm{c}_{2} \geq \cdots \geq \mathrm{c}_{j} \geq \cdots \geq \mathrm{c}_{n-1} \geq \mathrm{c}_{n} .
$$

The total number of sequences of this type for the distributions of the system (3) is determined by the number of permutations $n$ ! In case when $n=3$, the field of distributions of the coefficients of relative importance degenerates into a right triangle with unitary legs. The number of subsets, each corresponding to its own ratio between the coefficients of relative importance of the indicators, is $P_{3}=3 !=6$ (for $n=4$, $P_{4}=4 !=24$, etc.).
The foregoing allows us to formulate an algorithm for finding the optimal value $D_{i}$ for each of the solution variants as follows:

1. The values of the relative importance indicators $C_{j}$ are ordered in the form of a sequence (5).

2 . For each comparison variant $i$, the linear programming problem is solved:

$$
\left\{\begin{array}{c}
d_{i}=\sum_{j=1}^{n} b_{i j} \mathrm{c}_{j} \rightarrow \max , \\
\sum_{j=1}^{n} \mathrm{c}_{j}=1,0 \leq \mathrm{c}_{j} \leq 1, \mathrm{c}_{j} \geq \mathrm{c}_{j+1}, j=\overline{1, n-1} .
\end{array}\right.
$$

The task has an analytical solution, in accordance with which the values of the coefficients of relative importance are determined [15]:

$$
\mathrm{c}_{j}=\left\{\begin{array}{l}
\frac{1}{k}, \text { or } j \leq k, \\
0, \text { or } j>k
\end{array},\right.
$$

if where the index $k$ is determined from the condition:

$$
b_{k j}=\max _{j} b_{i j} \text {. }
$$

3. Next, the efficiency values for each solution are calculated:

$$
d_{i}=\sum_{j=1}^{n} b_{i j} c_{j}
$$

and the optimal type of vehicle $f$ is determined from the relation:

$$
D_{f}=\max _{1 \leq i \leq m} d_{i}
$$

This algorithm allows you to create a method for determining the weight coefficients and searching for the optimal solution in a system of multi-criteria evaluation of individual properties of a vehicle [16].

\section{FINDINGS}

The key advantages of the developed method in comparison with those currently used are:

1. The absence of a formalized relationship between the coefficients of relative importance of certain vehicle quality criteria.

2. In order to obtain a solution of the task connected with evaluating the quality of a vehicle using several efficiency criteria, it is sufficient to establish the order of priority between the established criteria.

3. The solution obtained is the highest possible in quantitative estimation, taking into account the performance indicators according to the established quality criteria.

4. The apparatus for obtaining the weight of the criterion is formalized, that is, it is objective in terms of obtaining the weight of a separate criterion for each individual solution.

The effectiveness of the developed method for solving this problem is proved by comparing the results of its application and the existing analog methods: the Laplace criterion, the 
Wald criterion, the Savage criterion, the Hurwitz criterion, the Fishburn estimate, etc. [15].

The scope of the developed method of obtaining an evaluation of the effectiveness of the subject of research by several criteria is quite wide. This approach allows us to obtain optimal results for a wide range of problems in decision-making theory:

1) vector optimization;

2) multi-criteria tasks;

3) tasks in which the solution is sought on the basis of expert assessments;

4) tasks based on the idea of ranking;

5) the problem of optimal allocation of resources for objects of various importance;

6) the problem of optimal distribution of homogeneous or dissimilar resources in conditions of incomplete information;

7) parametric programming tasks;

8) the task of determining the optimal structure of the required resources, etc.

It is obvious that the listed tasks are relevant for managing the state of the vehicle's quality at all stages of its lifecycle in the specific conditions of operation of the AIC [17], and the developed method makes it possible to deduce the removal of uncertainty and clarification of information on the probabilities of the conditions of the vehicle's operating environment to a new qualitative level.

\section{CONCLUSION}

A cardinal solution to the problem of the efficient use of road transport by the agro-industrial complex is organically connected with the improvement of the structure of transport parks of agricultural enterprises. The formation of automobile parks should be carried out taking into account the specific features of agricultural production, the technology of transportation processes, as well as the features of vehicle designs that determine their technological adaptation to the full use of their operational parameters in these conditions.

The presented method for solving multi-criteria tasks allows us to evaluate the quality of vehicles used in the agro-industrial complex taking into account the current environmental requirements: ensuring the environmental, constructive and fire safety of a vehicle. The solution of this task will make it possible to increase the efficiency and safety of the use of agroindustrial complex transport parks by fully realizing the potential capabilities of each transport unit as well as the entire fleet, increasing the possibility of mastering peak volumes of traffic by a smaller number of vehicles, reducing the need for attracted transport. In turn, this will entail a reduction in the share of transportation costs in total production costs for agricultural production and will result in significant cost savings. The transport parks formed on the basis of the presented method will meet the requirements of the conditions for the production of agricultural enterprises both in general and individual elements (vehicles).

\section{References}

[1] E.V. Nochevkina, L.V. Goryainova, "Technical equipment of agriculture in Russia", Scientific community of students of the XXI century. Economics: collection of articles. Art. by materials of the XXXVIII International stud. scientific-practical Conference, vol. 1 (38), pp. 274 279, 2016. URL: http://sibac.info/archive/economy/1(38).pdf (reference date is June 8, 2018).

[2] S.M. Moroz, Methods of providing operational condition of vehicles, Moscow: MADI, 2015, 204 p.

[3] B.D. Prudovsky, Management of technical operation of cars by normative indicators, Moscow: Transport, 1990, 239 p.

[4] E.S. Kuznecov, Management of technical systems, Moscow: MADI, 2003, 247 p.

[5] A.A. Kapustin, «Automobile-road transport, a dangerous stage of development.», Collected materials of the 74th Conference of MADI, Moscow: MADI, 2016, p. $224-228$.

[6] Methods of evaluation and selection of trucks. [Electronic resource]:Access mode: http://pandia.ru/889360/

[7] Methods for assessing the efficiency of all-wheel drive vehicles, Bronnitsy, 2005, 144 p.

[8] I.A. Pliyev, A.N. Verzhbitsky, «Methodology for assessing the technical level of multipurpose automatic telephone exchanges», Automobile industry, vol. 11, 1999, p. 34-36.

[9] Kh.A. Faskhiev, A.V. Krakhmaleva, «Assessment of the level of competitiveness of trucks and their engines», Marketing in Russia and abroad, vol. 5, 2004, p. 3-16.

[10] A.V. Parkhomenko, L.V. Parkhomenko, B.I. Gerasimov, Economic and mathematical models of controlling in an industrial enterprise, Tambov: Publishing house of Tambov state tech. University, 2005, 96 p.

[11] T. Saati, Decision Making. Method of hierarchy analysis, Moscow: Radio and communication, $1993,278 \mathrm{p}$.

[12] A.N. Tikhomirova, E.V. Sidorenko, «Modification of the method of hierarchy analysis by T. Saati for calculation of the weights of the criteria for the evaluation of innovative projects», Modern problems of science and education, vol. 2, 2012.

[13] E.Mushik, P. Mueller, Methods of making technical decisions, Translation from German, Moscow: Mir, 1990, 208 p.

[14] B.D. Prudovsky, Methods for solving multi-criteria transportation tasks, Bulletin of Civil Engineers, vol. 2(49), Saint-Petersburg: SPSUACE, 2015, p.154-159.

[15] M.Yu. Karelina, I.V. Arifullin, A.V. Terentyev, «Analytical definition of weight coefficients for multi-criteria evaluation of vehicle efficiency», Bulletin of the Moscow Automobile and Road State Technical University (MADI), vol. 1 (52), 2018, p. 3-9.

[16] A.V. Terentyev, B.D. Prudovsky, «Investigation methods for «current repairs labour-intensiveness» factor for a vehicle», Life Science Journal, vol. 11 (10s), 2014, p. 307-310.

[17] S.A. Evtyukov, A.V. Terentyev, G. Ginsburg, «Methodology for managing the rational lifetime of a vehicle», The world of transport and technological machines, vol. 1 (56), 2017, p. 3-10. 